\title{
Prevalência da INGestão de Álcool nos Adolescentes - Estudo PINGA
}

André Reis, ${ }^{1}$ Joana Barros, ${ }^{2}$ Cristina Fonseca, ${ }^{3}$ Luísa Parreira, ${ }^{4}$ Manuel Gomes, ${ }^{5}$ Ivo Figueiredo, ${ }^{6}$ Sandra Matapa ${ }^{7}$

\section{RESUMO}

Objectivos: Caracterizar o consumo de álcool nos adolescentes, verificar a sua relação com sexo e idade e determinar os motivos de consumo.

Tipo de estudo: Observacional, transversal e analítico.

Local: Escolas do 3. ciclo/secundárias do distrito de Bragança.

População em estudo: Jovens dos 13 aos 19 anos a frequentar as referidas escolas.

Métodos: Adolescentes seleccionados por amostragem aleatória (etapas múltiplas). Dimensão calculada da amostra: 955 indivíduos (para prevalência esperada: 45\%; nível de precisão: 3,0\%; IC: 95\%). Variáveis: sexo; idade; consumo de álcool e motivos de consumo. Dados recolhidos por questionário validado (AAIS; Pontuação: 0-79), tratados pelo teste do qui-quadrado ( $\alpha=0,05$ ) utilizando software Excel ${ }^{\circledR}$ e SPSS ${ }^{\circledR}$.

Resultados: Obtiveram-se 1061 inquéritos validados, 51,6\% do sexo feminino; 18,5\% com 13-14 anos, 59,9\% com 15-17 anos e $21,7 \%$ com $18-19$ anos. Quanto ao consumo, $89,8 \%$ já experimentaram álcool, dos quais $96,4 \%$ no último ano e $69,5 \%$ dos jovens apresentam pelo menos consumo mensal. Destes, 226 bebem todos os fins-de-semana e 21 diariamente; $27,7 \%$ praticam «binge drinking». Verificou-se que $68,3 \%$ dos jovens apresentam consumo de risco. Relacionando o sexo com o consumo de álcool, verificou-se que o sexo masculino apresenta maior consumo $(p=0,032)$. Na amostra obtida, o grupo etário dos 18-19 anos é aquele que apresenta um consumo mais elevado. Relativamente aos motivos de consumo, destacam-se o acompanhar os amigos (coping) e o paladar, independentemente do sexo e grupo etário.

Conclusões: $O$ consumo de álcool encontrado nesta faixa etária é elevado e superior à literatura, existindo um predomínio do sexo masculino. O meio social assume-se como um factor determinante, nomeadamente o consumo para acompanhar os amigos e assim corresponder às expectativas do grupo. Dados os resultados obtidos, torna-se fundamental o desenvolvimento de estratégias de intervenção a este nível.

Palavras-chave: Consumo de Bebidas Alcoólicas; Etanol; Adolescente; Prevalência.

\section{INTRODUÇÃO}

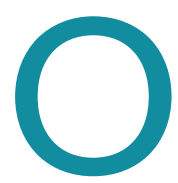

álcool é a substância de abuso mais comercializada a nível mundial ${ }^{1}$ e uma das poucas substâncias psicotrópicas que tem o seu consumo legalizado e inclusivamente incentivado pela sociedade. ${ }^{2}$ De acordo com a

\footnotetext{
${ }^{1}$ Assistente de MGF - US Santa Maria (ACES do Nordeste)

${ }^{2}$ Assistente de MGF - USF Santa Luzia (ACES Tâmega III - Vale do Sousa Norte)

${ }^{3}$ Assistente de MGF - UCSP Mirandela I (ACES do Nordeste)

${ }^{4}$ Assistente de MGF - UCSP Miranda do Douro (ACES do Nordeste)

${ }^{5}$ Interno de MGF - US Santa Maria (ACES do Nordeste)

${ }^{6}$ Interno de MGF - UCSP Mirandela I (ACES do Nordeste)

${ }^{7}$ Interna de MGF - UCSP Macedo de Cavaleiros (ACES do Nordeste)
}

Organização Mundial de Saúde (OMS), o seu consumo pode ser classificado em cinco categorias: ${ }^{3}$ abstinência, consumo não nocivo, consumo de risco, consumo nocivo e dependência (Quadro I).

Nos adolescentes o consumo de álcool tem vindo a assumir proporções alarmantes, tratando-se da principal substância de abuso nesta faixa etária. ${ }^{5}$ Um padrão de consumo que tem assumido importância crescente neste grupo é o chamado «binge drinking», que corresponde ao consumo de cinco ou mais bebidas contendo álcool (quatro no sexo feminino), num período de poucas horas, geralmente com o objectivo de atingir a embriaguez. ${ }^{6,7}$ 
O consumo excessivo de álcool tem consequências negativas imediatas para a saúde, ${ }^{7,8}$ podendo causar danos cerebrais, interferindo com o desenvolvimento mental e com o aproveitamento escolar. Encontra-se frequentemente relacionado com comportamentos anti-sociais, violência, suicídio, acidentes de viação, comportamentos sexuais de risco, delinquência juvenil e abuso concomitante de outras substâncias..$^{2,5,7,8,10-12}$ Está também comprovadamente associado a um risco aumentado de desenvolvimento de doença hepática, hipertensão arterial, doença coronária, acidentes vasculares cerebrais e cancro da mama, boca ou esófago, entre outras consequências. ${ }^{2,4,13} \mathrm{Um}$ aspecto particularmente preocupante é o facto de o consumo de álcool ter início em idades cada vez mais precoces, ${ }^{5,12,14}$ o que aumenta o risco de desenvolvimento de problemas no futuro. ${ }^{15,16}$

Segundo um estudo realizado nos EUA em 2003, o consumo de álcool no último mês em jovens entre os 12 e os 20 anos foi

QUADRO I. Classificação do padrão de consumo de álcool de acordo com a OMS.

\begin{tabular}{ll} 
Categoria de Consumo & \multicolumn{1}{c}{ Descrição } \\
\hline Abstinência & Ausência total de consumo de bebidas alcoólicas. \\
\hline Consumo não nocivo & $\begin{array}{l}\text { Consumo que não ultrapassa as } 20 \mathrm{~g} \text { de álcool/dia (2 } \\
\text { UBP), existindo pelo menos dois dias semanais sem } \\
\text { consumo. }\end{array}$ \\
\hline Consumo de risco & $\begin{array}{l}\text { Padrão de consumo do qual pode vir a resultar dano } \\
\text { físico ou psíquico, se persistir. }\end{array}$ \\
\hline Pansumo nocivo & $\begin{array}{l}\text { Padrão de consumo que causa danos físicos e } \\
\text { psíquicos, }{ }^{4} \text { mas não preenche os critérios de }\end{array}$ \\
\hline dependência.
\end{tabular}

Unidade de Bebida Padrão (UBP): Conceito criado para quantificar o consumo de álcool que corresponde, em Portugal, a 10-12 g de álcool, ou seja, $3 \mathrm{dL}$ de cerveja, $1,65 \mathrm{dL}$ de vinho ou $0,5 \mathrm{dL}$ de aguardente, entre outros. ${ }^{2}$ de $50-52,8 \% ;{ }^{17} 30 \%$ referiam praticar «binge drinking» pelo menos uma vez por mês e $58 \%$ dos alunos do $12 .^{\circ}$ ano e $20 \%$ dos alunos do $8 .^{\circ}$ ano referiam já ter ficado embriagados pelo menos uma vez na vida. ${ }^{8}$ De acordo com um outro estudo de grande dimensão, $25,2 \%$ dos estudantes do $7 .^{\circ}$ ao $12 .^{\circ}$ ano tinham consumido pelo menos uma bebida no último mês. ${ }^{14}$

Em Portugal, um estudo realizado no Porto com alunos do $9 .^{\circ}$ ao $12 .{ }^{\circ}$ ano de escolaridade,$^{18}$ revelou que a proporção de consumo de álcool neste grupo era de $59,8 \%$. Um outro estudo, ${ }^{19}$ realizado a nível nacional com alunos do $6 .^{\circ}$ ao $10 .^{\circ}$ ano, demonstrou que $55,6 \%$ dos jovens do sexo masculino e $48,8 \%$ do sexo feminino já haviam experimentado bebidas alcoólicas, enquanto $44 \%$ admitiam consumir ocasionalmente e $7,1 \%$ faziam-no regularmente.

Parecem ser várias as razões que motivam o consumo de álcool pelos adolescentes, nomeadamente o gosto pelo paladar, a necessidade de se integrarem no grupo de amigos (como estratégia de coping), de se sentirem adultos ou o desejo de relaxar e esquecer os pro- blemas. Dois estudos realizados neste âmbito ${ }^{20-21}$ concluíram que o consumo de álcool nos adolescentes ocorria sobretudo por motivos sociais, nomeadamente como estratégia de coping. No entanto, um número significativo de jovens consumia álcool com o objectivo de se embriagar. ${ }^{21}$

Dada a escassez de estudos sobre o consumo de álcool nos adolescentes do Nordeste Transmontano, região tradicionalmente referida por algum excesso no consumo, a determinação da proporção de adolescentes que consomem bebidas alcoólicas de forma regular e a quantificação e caracterização desse consumo permitirá adequar estratégias de intervenção.

\section{OBJECTIVOS}

O presente estudo tem como objectivos caracterizar o consumo de álcool nos adolescentes e verificar se existe relação com o sexo e com a idade. Pretende-se ainda determinar as principais razões que motivam esse consumo, verificando também se estão relacionadas com o sexo e com a idade. 


\section{MATERIAL E MÉTODOS}

Tipo de Estudo: Observacional, transversal e analítico.

Local do estudo: Escolas do $3 .^{\circ}$ ciclo e secundárias do distrito de Bragança.

População em estudo: Constituída pelos 14.826 adolescentes a frequentar as referidas escolas no ano lectivo $2008 / 2009,{ }^{18}$ definindo-se como adolescentes os jovens com idades compreendidas entre os 13 e os 19 anos.

Critérios de exclusão: adolescentes ausentes da respectiva escola durante o período de recolha de dados e estudantes dos estabelecimentos de ensino seleccionados com menos de 13 anos ou mais de 19 anos.

Caracterização da amostra e técnica de amostragem: Amostra constituída por adolescentes de turmas e escolas do distrito de Bragança no ano de 2009, seleccionadas através de uma técnica de amostragem aleatória por etapas múltiplas, seleccionando inicialmente as escolas e posteriormente algumas turmas dentro de cada escola. A dimensão calculada da amostra, para uma prevalência esperada de $45 \%$, com um nível de precisão de $3,0 \%$ e um intervalo de confiança de 95\%, corresponde a 955 indivíduos.

\section{Variáveis em estudo:}

- Sexo: género masculino ou feminino.

- Idade: número de anos de vida. Agrupada em três intervalos: 13-14 anos; 15-17 anos e 18-19 anos.

- Consumo de álcool: consumo de bebidas contendo álcool. Agrupado em cinco categorias: abstinente (0 pontos); bebedor ocasional (1-19 pontos); consumo de risco (20-41 pontos); consumo nocivo (42-57 pontos) e dependência (58-79 pontos).

- Motivos de consumo: classificados nas seguintes categorias - gosto pelo paladar; para acompanhar os amigos; para se sentir adulto; por nervosismo/tensão/problemas ou ainda por tristeza, solidão ou pena de si próprio.

\section{Métodos de recolha de informação:}

Dados recolhidos através de um questionário internacional validado para a população portuguesa, anónimo e de auto-preenchimento, distribuído nas escolas e turmas seleccionadas. Como forma de garantir o anonimato, após o seu preenchimento, os questioná- rios foram colocados em envelopes fechados.

O «Adolescent Alcohol Involvement Scale» (AAIS) é um questionário de 14 questões, dirigido especificamente para populações jovens. ${ }^{23} \mathrm{~A}$ pontuação total varia de 0 a 79 pontos. A cada alínea a) corresponde um ponto (excepto nas questões 1, 2, 6, 12, 13 e 14, em que corresponde a 0 pontos), a cada alínea b) dois pontos e assim sucessivamente. Em cada uma das questões pode ser seleccionada mais do que uma alínea, contudo, para efeitos de pontuação, apenas é contabilizada a resposta de maior pontuação. Os resultados encontram-se agrupados em 5 categorias: abstinência ( 0 pontos); consumo ocasional (1-19 pontos); consumo de risco (2041 pontos); consumo nocivo (42-57 pontos) e dependência (58-79 pontos). Em Portugal, Barrias $e t a l^{4}$ traduziram e validaram este questionário, aplicando-o a amostras populacionais de várias regiões.

\section{Tratamento dos dados:}

Dados codificados e registados em base de dados informática (Microsoft Excel ${ }^{\circledR}$ ). Para o tratamento estatístico, utilizou-se o programa SPSS v.13.0 recorrendose ao teste do qui-quadrado e adoptando um nível de significância de 0,05.

\section{RESULTADOS}

Foram obtidos 1343 inquéritos, dos quais 240 foram excluídos por se encontrarem incorrectamente preenchidos ou incompletos, o que corresponde a uma taxa de resposta de $82,1 \%$. Destes, foram ainda excluídos mais 42 , por não preencherem os critérios de inclusão, restando assim um total de 1061 inquéritos válidos e correctamente preenchidos. A caracterização da amostra obtida encontra-se no Quadro II. A média de idades foi de 16 anos e a mediana de 16,1 anos.

Relativamente à proporção de consumo de álcool, a frequência verificada foi elevada, com $69,5 \%$ dos jovens a apresentarem pelo menos um consumo mensal de bebidas alcoólicas (Quadro III).

Dos 1061 jovens que responderam ao questionário, 89,8\% (953) já consumiram álcool em alguma ocasião, $96,4 \%$ dos quais no último ano. Apenas $10,2 \%$ referem nunca ter ingerido bebidas alcoólicas, enquanto 21,3\% dizem beber todos os fins-de-semana e 2,0\% diariamente. Por outro lado, 27,7\% (294) dos jovens dizem praticar «Binge Drinking». 


\begin{tabular}{|lccc|}
\multicolumn{4}{|c}{ QUADRO II. Caracterização da amostra } \\
$\mathbf{n}=1061$ & & $\mathbf{n}$ & $\%$ \\
\hline Sexo & M & 514 & 48,4 \\
\hline Idade & $\mathbf{F}$ & 547 & 51,6 \\
\hline & $13-14$ & 196 & 18,5 \\
\hline & $15-17$ & 635 & 59,9 \\
\hline & $18-19$ & 230 & 21,7 \\
\hline
\end{tabular}

álcool, tendo sido referida por $36,1 \%$ dos jovens. Contudo, uma percentagem de aproximadamente $6 \%$ admitiu ter começado a beber antes dos 10 anos.

Relativamente ao padrão de consumo de álcool obtido, mais de dois terços dos adolescentes apresentam um consumo de risco. O Quadro IV apresenta esses resultados.

Relacionando o sexo com o consumo de álcool, verificou-se que existe uma relação estatisticamente sig-

\begin{tabular}{|c|c|c|c|c|c|c|c|c|c|c|c|c|}
\hline \multirow{3}{*}{$\begin{array}{l}\text { Frequência de } \\
\text { Consumo }\end{array}$} & \multirow{2}{*}{\multicolumn{2}{|c|}{ Total }} & \multicolumn{4}{|c|}{ Sexo } & \multicolumn{6}{|c|}{ Idade } \\
\hline & & & \multicolumn{2}{|c|}{$\mathbf{F}$} & \multicolumn{2}{|c|}{$M$} & \multicolumn{2}{|c|}{$13-14$} & \multicolumn{2}{|c|}{$15-17$} & \multicolumn{2}{|c|}{ 18-19 } \\
\hline & $\mathrm{n}$ & $\%$ & $\mathrm{n}$ & $\%$ & $\mathrm{n}$ & $\%$ & $\mathrm{n}$ & $\%$ & $n$ & $\%$ & $\mathrm{n}$ & $\%$ \\
\hline Nunca & 108 & 10,2 & 64 & 59,3 & 44 & 40,7 & 57 & 52,8 & 42 & 38,9 & 9 & 8,3 \\
\hline $1-2 \times /$ Ano & 216 & 20,4 & 132 & 61,1 & 84 & 38,9 & 52 & 24,1 & 131 & 60,7 & 33 & 15,3 \\
\hline 1-2 x / Mês & 435 & 41,0 & 260 & 59,8 & 175 & 40,2 & 69 & 15,9 & 268 & 61,6 & 98 & 22,5 \\
\hline Todos os FDS & 226 & 21,3 & 72 & 31,9 & 154 & 68,1 & 9 & 4,0 & 150 & 66,4 & 67 & 29,7 \\
\hline Várias x / Semana & 55 & 5,2 & 14 & 25,5 & 41 & 74,6 & 5 & 9,1 & 33 & 60,0 & 17 & 30,9 \\
\hline Diariamente & 21 & 2,0 & 5 & 23,8 & 16 & 76,2 & 4 & 19,1 & 11 & 52,4 & 6 & 28,6 \\
\hline
\end{tabular}

\begin{tabular}{|c|c|c|}
\hline \multicolumn{3}{|c|}{$\begin{array}{l}\text { QUADRO IV. Padrão de consumo de } \\
\text { álcool obtido }\end{array}$} \\
\hline$n=1061$ & $\mathbf{n}$ & $\%$ \\
\hline Abstinência & 108 & 10,2 \\
\hline Consumo Ocasional & 15 & 1,4 \\
\hline Consumo de Risco & 725 & 68,3 \\
\hline Consumo Nocivo & 195 & 18,4 \\
\hline Dependência & 18 & 1,7 \\
\hline
\end{tabular}

Constatou-se ainda que o intervalo 14-15 anos foi a idade mais frequente para o início do consumo de nificativa, sendo que o sexo masculino apresenta maior consumo (pontuação média: 34,3 , que corresponde a um consumo de risco; $p=0,032$ ), como se pode comprovar no Quadro V.

Analisando a distribuição do consumo de álcool por intervalos de idade, verificou-se que este aumenta com a idade, apesar de esta relação não ser estatisticamente significativa (Quadro VI).

Relativamente aos motivos de consumo apontados, estes encontram-se expressos no Quadro VII, destacando-se o "acompanhar os amigos» (coping) e o "gosto pelo paladar» como principais motivos.

\begin{tabular}{|c|c|c|c|c|c|c|c|c|c|c|c|}
\hline \multirow[t]{2}{*}{ Sexo } & \multicolumn{2}{|c|}{ Abstinência } & \multicolumn{2}{|c|}{$\begin{array}{l}\text { Consumo } \\
\text { Ocasional }\end{array}$} & \multicolumn{2}{|c|}{$\begin{array}{r}\text { Consumo } \\
\text { de Risco }\end{array}$} & \multicolumn{2}{|c|}{$\begin{array}{r}\text { Consumo } \\
\text { Nocivo }\end{array}$} & \multicolumn{2}{|c|}{ Dependência } & \multirow[t]{2}{*}{$\mathbf{P}$} \\
\hline & $\mathbf{n}$ & $\%$ & $\mathbf{n}$ & $\%$ & n & $\%$ & $\mathbf{n}$ & $\%$ & $n$ & $\%$ & \\
\hline M & 44 & 8,6 & 7 & 1,4 & 341 & 66,3 & 112 & 21,8 & 10 & 1,9 & 0,032 \\
\hline $\mathbf{F}$ & 64 & 11,7 & 8 & 1,5 & 384 & 70,2 & 83 & 15,2 & 8 & 1,5 & \\
\hline
\end{tabular}




\begin{tabular}{|c|c|c|c|c|c|c|c|c|c|c|c|}
\hline \multirow[t]{2}{*}{$\begin{array}{l}\text { Idade } \\
\text { (anos) }\end{array}$} & \multicolumn{2}{|c|}{ Abstinência } & \multicolumn{2}{|c|}{ Ocasional } & \multicolumn{2}{|c|}{$\begin{array}{c}\text { Consumo } \\
\text { de Risco }\end{array}$} & \multicolumn{2}{|c|}{$\begin{array}{c}\text { Consumo } \\
\text { nocivo }\end{array}$} & \multicolumn{2}{|c|}{ Dependência } & \multirow[t]{2}{*}{$\mathbf{P}$} \\
\hline & $\mathbf{n}$ & $\%$ & $\mathbf{n}$ & $\%$ & $\mathbf{n}$ & $\%$ & $\mathbf{n}$ & $\%$ & $\mathrm{n}$ & $\%$ & \\
\hline $13-14$ & 57 & 29,1 & 8 & 4,1 & 117 & 59,2 & 12 & 6,1 & 4 & 1,5 & \\
\hline $15-17$ & 42 & 6,6 & 6 & 0,9 & 449 & 70,6 & 129 & 20,3 & 10 & 1,6 & $>0,05$ \\
\hline 18-19 & 9 & 3,9 & 1 & 0,4 & 159 & 70,0 & 54 & 23,5 & 4 & 2,2 & \\
\hline
\end{tabular}

Verificou-se a existência de uma relação entre sexo e motivos de consumo, embora não estatisticamente significativa. Em ambos os sexos destacam-se como motivos mais frequentes «acompanhar os amigos» $\mathrm{e}$ «gosto pelo paladar». No entanto, no sexo masculino estes predominam sensivelmente de forma equitativa, enquanto no sexo feminino predomina claramente o «acompanhar os amigos» (Quadro VIII).

Analisando os motivos de consumo por grupo etário, não foi encontrada qualquer relação - independentemente do grupo etário, os motivos mais referidos foram sempre «acompanhar os amigos» e «gosto pelo paladar» (Quadro IX).

\begin{tabular}{lrc}
\multicolumn{3}{l}{ QUADRO VII. Motivos de consumo de álcool obtidos } \\
\hline $\mathrm{n}=1061$ & $\mathrm{n}$ & $\%$ \\
\hline Gosto pelo paladar & 353 & 33,3 \\
\hline Acompanhar amigos & 527 & 49,7 \\
\hline Sentir-se adulto & 15 & 1,4 \\
\hline Nervosismo, tensão ou problemas & 36 & 3,4 \\
\hline Tristeza, solidão, pena de si próprio & 22 & 2,1 \\
\hline Nunca beberam & 108 & 10,2
\end{tabular}

\section{DISCUSSÃO}

A proporção de consumo de álcool nesta faixa etária é elevada e superior à obtida noutros estudos realizados neste âmbito, ${ }^{5,7,14,18}$ o que levanta a hipótese de o Nordeste transmontano ser efectivamente uma região com um consumo de álcool nos adolescentes acima da média. Um outro dado que sustenta esta afirmação é a elevada proporção de jovens que já consumiram álcool em alguma ocasião $(89,8 \%)$, valor bastante acima daquele encontrado na literatura para esta faixa etária. No que diz respeito ao «binge drinking», a taxa encontrada foi semelhante à obtida no estudo realizado nos EUA em $2003 .{ }^{17}$ Este aspecto é particularmente relevante, na medida em que este tipo de consumo demonstrou estar associado a maior dano social e para a saúde. ${ }^{11}$

O padrão de consumo predominante foi o consumo de risco, seguido pelo consumo nocivo, constatando-se assim a existência de padrões de consumo preocupantes. Verificou-se um predomínio de consumo no sexo masculino. Existe também um predomínio de consumo nas faixas etárias mais elevadas, ainda que não estatisticamente significativo. Ambos os resultados vão, contudo, de encontro ao referido na literatura. ${ }^{14}$

Em relação aos motivos de consumo, os motivos de carácter social assumem-se como um factor determi-

\begin{tabular}{|c|c|c|c|c|c|c|c|c|c|c|c|}
\hline \multirow[t]{2}{*}{ Sexo } & \multicolumn{2}{|c|}{$\begin{array}{c}\text { Gosto pelo } \\
\text { Paladar }\end{array}$} & \multicolumn{2}{|c|}{$\begin{array}{c}\text { Acompanhar } \\
\text { Amigos }\end{array}$} & \multicolumn{2}{|c|}{$\begin{array}{c}\text { Sentir-se } \\
\text { Adulto }\end{array}$} & \multicolumn{2}{|c|}{$\begin{array}{c}\text { Nervosismo, } \\
\text { Tensão ou } \\
\text { Problemas }\end{array}$} & \multicolumn{2}{|c|}{$\begin{array}{c}\text { Tristeza, Solidão, } \\
\text { Pena de si } \\
\text { próprio }\end{array}$} & \multirow[t]{2}{*}{ P } \\
\hline & $n$ & $\%$ & $n$ & $\%$ & $n$ & $\%$ & $n$ & $\%$ & $n$ & $\%$ & \\
\hline$M$ & 205 & 39,9 & 224 & 43,6 & 11 & 2,1 & 18 & 3,5 & 12 & 2,3 & $>0,05$ \\
\hline$F$ & 148 & 27,1 & 303 & 55,4 & 4 & 0,7 & 18 & 3,3 & 10 & 1,8 & \\
\hline
\end{tabular}


QUADRO IX. Motivos de consumo por grupo etário

\begin{tabular}{|c|c|c|c|c|c|c|c|c|c|c|c|}
\hline \multirow[t]{2}{*}{$\begin{array}{l}\text { Idade } \\
\text { (anos) }\end{array}$} & \multicolumn{2}{|c|}{$\begin{array}{c}\text { Gosto pelo } \\
\text { Paladar }\end{array}$} & \multicolumn{2}{|c|}{$\begin{array}{c}\text { Acompanhar } \\
\text { Amigos }\end{array}$} & \multicolumn{2}{|c|}{$\begin{array}{l}\text { Sentir-se } \\
\text { Adulto }\end{array}$} & \multicolumn{2}{|c|}{$\begin{array}{c}\text { Nervosismo, } \\
\text { Tensão ou } \\
\text { Problemas }\end{array}$} & \multicolumn{2}{|c|}{$\begin{array}{c}\text { Tristeza, Solidão, } \\
\text { Pena de si } \\
\text { próprio }\end{array}$} & \multirow[b]{3}{*}{$>0,05$} \\
\hline & $n$ & $\%$ & $n$ & $\%$ & $\mathrm{n}$ & $\%$ & $n$ & $\%$ & $n$ & $\%$ & \\
\hline $13-14$ & 51 & 25,9 & 74 & 37,8 & 6 & 3,1 & 5 & 2,6 & 3 & 1,5 & \\
\hline $15-17$ & 227 & 35,8 & 324 & 51,0 & 6 & 0,9 & 23 & 3,6 & 13 & 2,1 & \\
\hline 18-19 & 75 & 32,6 & 129 & 56,1 & 3 & 1,3 & 8 & 3,5 & 6 & 2,6 & \\
\hline
\end{tabular}

nante, à semelhança do referido noutros estudos, ${ }^{20,21}$ independentemente do sexo ou da idade. Assim, o gosto pelo paladar e o acompanhar os amigos (motivos baseados num reforço positivo) foram os mais frequentemente apontados. Este facto poderá estar associado a um esforço, por parte dos adolescentes, para corresponder às expectativas do grupo, para se sentirem integrados e respeitados.

É também preocupante a elevada percentagem de jovens com início de consumo de álcool muito precoce, já que quase metade dos jovens referiram começar a beber antes dos 15 anos. Constitui ainda motivo de reflexão a importante percentagem dos que iniciaram o consumo antes dos 10 anos de idade. Uma das razões para este início de consumo tão precoce é o facto de as bebidas alcoólicas funcionarem como uma droga socialmente aceite e facilmente acessível, apesar de a sua venda estar interdita a menores de 16 anos. ${ }^{25}$

No que diz respeito à validade interna deste estudo, pode ser caracterizada como boa, uma vez que a amostra foi representativa e aleatória e se obteve uma boa taxa de adesão. Relativamente a limitações, admite-se a existência de alguns viéses, nomeadamente de memória ou de medição, apesar da garantia de anonimato e confidencialidade. Quanto à validade externa, os resultados obtidos foram semelhantes a outros estudos existentes, embora com uma proporção de consumo ligeiramente superior, o que reforça a ideia de estarmos perante um problema da maior gravidade.

Este estudo permitiu assim caracterizar o consumo de álcool dos adolescentes do distrito de Bragança, possibilitando o estabelecimento de estratégias de prevenção e educação mais direccionadas, nomeadamente a divulgação destes resultados nas respectivas esco- las e realização de sessões educativas. Uma outra medida a implementar seria a abordagem desta problemática nos Exames Globais de Saúde dos 11-13 anos, sensibilizando desde logo os jovens e encarregados de educação.

Como linhas futuras de investigação sugere-se a avaliação de possíveis factores relacionados com o consumo de álcool, nomeadamente de natureza psicológica ou social. Seria igualmente pertinente verificar se existe relação entre motivos de consumo, quantidade consumida e consequências directas desse consumo.

\section{REFERÊNCIAS BIBLIOGRÁFICAS}

1. Advisory Council on the Misuse of Drugs; 2006.

2. Mello ML, Barrias J, Breda J. Álcool e problemas ligados ao álcool em Portugal. Lisboa: DGS; 2001.

3. World Health Organization. Lexicon of Alcohol and Drug Terms. Geneve:WHO; 1994.

4. Reid MC, Fiellin DA, O'Connor PG. Hazardous and harmful alcohol consumption in primary care. Arch Intern Med 1999 Aug 9-23; 159 (15): 1681-9.

5. Moreno RS, Ventura RN, Brêtas JR. O uso de álcool e tabaco por adolescentes do município de Embu, São Paulo, Brasil. Rev Esc Enferm USP 2010 Dec; 44 (4): 969-77.

6. Levy DT, Miller TR, Cox K. Costs of Underage Drinking. Calverton, MD: Pacific Institute for Research and Evaluation; 1999.

7. Vieira DL, Ribeiro M, Romano M, Laranjeira RR. Álcool e adolescentes: estudo para implementar políticas municipais. Rev Saúde Pública 2007 Jun; 41 (3): 396 403.

8. Kulig JW;American Academy of Pediatrics Committee on Substance Abuse. Tobacco, alcohol, and other drugs: the role of the pediatrician in prevention, identification, and management of substance abuse. Pediatrics 2005 Mar; 115 (3): 81621.

9. American Medical Association. Underage drinkers at higher risk of brain damage than adults. AMA Report. Chicago, IL: American Medical Association; 2002.

10. National Institute for Health and Clinical Excellence. Interventions in schools to prevent and reduce alcohol use among children and young people. London: NICE; 2007.

11. Karagülle D, Donath C, Grässel E, Bleich S, Hillemacher T. Rauschtrinken bei Jugendlichen und jungen Erwachsenen [Binge drinking in adolescents and young adults]. Fortschr Neurol Psyquiatr 2010 Apr; 78 (4): 196202.

12. Office of Applied Studies. Results from the 2001 National Household Survey 
on DrugAbuse.Volume 1: Summary of National Findings. Rockville, MD: US Dept of Health and Human Services, Substance Abuse and Mental Health Services Administration; 2002.

13. McCambridge J, McAlaney J, Rowe R. Adult consequences of late adolescent alcohol consumption: a systematic review of cohort studies. PLoS Med $2011 \mathrm{Feb}$ 8; 8 (2): e1000413. doi:10.1371/journal.pmed. 1000413.

14. Xing Y, Ji C, Zhang L. Relationship of binge drinking and other health compromising behaviour among urban adolescents in China. J Adolesc Health 2006 Oct; 39 (4): 495-500.

15. DeWit DJ, Adlaf EM, Offord DR, Ogborne AC. Age at first alcohol use: a risk factor for the development of alcohol disorders. Am J Psychiatry 2000 May; 157 (5): 74550.

16. Blomeyer D, Buchmann AF, Schmid B, Jennen-Steinmetz C, Schmidt MH, Banaschewski T, et al. Age at first drink moderates the impact of current stressful life events on drinking behaviour in young adults. Alcohol Clin Exp Res 2011 Jun; 35 (6): 1142-8. doi:10.1111/j.1530-0277.2011.01447.x.

17. Foster SE, Vaughan RD, Foster WH, Califano JA Jr. Alcohol consumption and expenditures for underage drinking and adult excessive drinking. JAMA $2003 \mathrm{Feb}$ 26; 289 (8): 989-95.

18. Ribeiro TT. Consumos de tabaco, álcool, haxixe e outras substâncias em meio escolar [Dissertação de Mestrado em Saúde Pública]. Porto: Faculdade de Medicina do Porto; 2000.

19. Matos MG, Simões C, Canha L, Fonseca S, Reis C.A Saúde dos Adolescentes Portugueses - Nacional e Região Norte 2002. Lisboa: Edições FMH; 2002.

20. FonteA,Alves A. Uso da escala de envolvimento com o álcool para adolescentes (AAIS): avaliação das características psicométricas. Rev Soc Port Alcoologia 1999 Out; 7 (4).
21. Steinhausen HC, Eschmann S, Heimgartner A, Metzke CW. Frequency, course and correlates of alcohol use from adolescence to young adulthood in a Swiss community survey. BMC Psychiatry 2008 Jan 117; 8: 5.

22. Instituto Nacional de Estatística.Anuário Estatístico da Região Norte 2009. Lisboa: Instituto Nacional de Estatística; 2010

23. Mayer J, Filstead WJ. The Adolescent Alcohol Involvement Scale: an instrument for measuring adolescents' use and misuse of alcohol. J Stud Alcohol 1979 Mar; 40(3): 291-300.

24. Barrias J, Neves G, Enes F, Pimentel L. A propósito de uma escala de envolvimento alcoólico no adolescente de John Mayer e William Filstead. Paper apresentado. In: II Encontro Nacional Sobre o Ambulatório em Saúde Mental, Setúbal; 1984.

25. Decreto-Lei n. ${ }^{\circ}$ 9/2002, de 24 de Janeiro.

\section{CONFLITOS DE INTERESSE}

Os autores declaram não possuir qualquer tipo conflitos de interesse

\section{ENDEREÇO PARA CORRESPONDÊNCIA}

André Reis

C.S. de Bragança - U.S. de Santa Maria

Av. Cidade de León, $s / n$

5300 Bragança

E-mail: andrexreis@hotmail.com

Recebido em 18/05/2010

Aceite para publicação em 16/06/2011

\section{ABSTRACT}

\section{ALCOHOL CONSUMPTION BY ADOLESCENTS - THE PINGA STUDY}

Aim: To characterize consumption of alcohol by adolescents, to study the association of alcohol consumption with gender and age and to determine the main reasons for consumption of alcohol by adolescents.

Type of study: Cross-sectional.

Location: High schools of the district of Bragança, Portugal.

Population: Teenagers aged 13 to 19 years old.

Methods: In the sample, $51.6 \%$ of the subjects were females, $18.5 \%$ were $13-14$ years old, $59.9 \%$ were $15-17$ years old and $21.7 \%$ were $18-19$ years old. The required sample size was 955 based on expected proportion of alcohol consumption of $45 \%$ with a $3 \%$ level of precision. Variables studied included gender, age, alcohol consumption and reasons for consumption. Data were collected using a validated questionnaire (AAIS) with scores ranging from 0-79. Associations between variables were tested using the with chi-square statistic with significance set at the 0.05 level.

Results: The authors obtained 1061 questionnaires.In the sample, $96.4 \%$ had consumed alcohol in the previous year; $69.5 \%$ of the subjects consumed alcohol at least monthly. 226 subjects drank every weekend and 21 drank daily; $27.7 \%$ practiced binge drinking. A risky pattern of alcohol consumption occurred in $68.3 \%$ of the adolescents. Consumption was higher among boys $(p=0,032)$ and increased with age. "Coping with peer pressure" and "taste" were given as the main reasons for drinking, independent of gender or age.

Conclusions: The proportion of adolescents consuming alcohol in this study was high compared to other published figures. This was higher among males and older studernts. The social environment is a strong determinant of drinking. In light of these results, an intervention strategy appears necessary in this population.

Keywords: Alcohol Drinking; Ethanol; Adolescent; Prevalence. 


\section{ANEXO I \\ QUESTIONÁRIO}

Por favor leia atentamente as seguintes questões e assinale a resposta apropriada. As suas respostas serão tratadas com a máxima confidencialidade. Em cada pergunta pode ser assinalada mais do que uma opção.

Idade: Sexo: Ano de escolaridade:

1. Com que frequência costuma tomar bebidas alcoólicas?

a) Nunca.

b) 1 ou 2 vezes por ano.

c) 1 ou 2 vezes por mês.

d) Todos os fins-de-semana.

e) Várias vezes por semana.

f) Todos os dias.

2. Quando bebeu pela última vez?

a) Nunca bebi.

b) Há mais de um ano.

c) Entre 6 meses e um ano.

d) Há várias semanas atrás.

e) A semana passada.

f) Ontem.

g) Hoje.

3. Habitualmente começo a beber:

a) Porque gosto do paladar.

b) Para acompanhar os amigos.

c) Para sentir-me como os adultos.

d) Porque me sinto nervoso, tenso, cheio de aborrecimento ou com problemas.

e) Porque me sinto triste, só, com pena de mim próprio.

4. Que bebida(s) costuma beber?

a) Vinho.

b) Cerveja.

c) Cocktails de bebidas alcoólicas.

d) Bagaço, brandy, whisky ou licores.

5. Como começou a beber?

a) Na presença dos pais ou parentes.

b) Com os irmãos ou irmãs.

c) Em casa sem os pais saberem.

d) Com os amigos.

e) Comprada por mim.
6. Com que idade bebeu pela primeira vez?

a) Nunca.

b) Recentemente.

c) Depois dos 15 anos.

d) Entre os 14 e os 15 anos.

e) Entre os 10 e os 13 anos.

f) Antes dos 10 anos.

\section{A que horas do dia costuma beber?}

a) Com as refeições.

b) À noite.

c) De tarde.

d) Normalmente de manhã ou com o pequeno-almoço.

e) Muitas vezes levanto-me durante a noite e bebo um copo.

8. Da primeira vez que bebeu, porque razão o fez?

a) Por curiosidade.

b) Porque me foi oferecido pelos pais ou família.

c) Entusiasmado pelos amigos.

d) Para me sentir mais adulto.

e) Para me embebedar ou para entrar «numa boa».

9. Quando bebe, quanto bebe?

a) 1 copo ou menos.

b) 2 copos.

c) 3 a 6 copos.

d) 6 ou mais copos.

e) Até ficar alegre ou bêbado.

10. Com quem costuma beber?

a) Só com os meus pais ou família.

b) Só com os meus irmãos ou irmãs.

c) Com os amigos da minha idade.

d) Com amigos ou companhias mais velhas.

e) Bebo sozinho.

11. Qual o efeito mais importante que já teve com a bebida?

a) Descontrair-me.

b) Ficar moderadamente alegre.

c) Ficar bêbado.

d) Ficar doente.

e) Desmaiar, perder os sentidos.

f) Beber muito e não me lembrar de nada no dia seguinte. 
12. Qual a maior consequência da bebida que já sentiu em toda a sua vida?

a) Nenhum - não senti qualquer efeito.

b) Interferiu com o que dizia.

c) Não me deixou passar um bom bocado.

d) Interferiu com o meu trabalho escolar.

e) Perdi alguns amigos por beber bebidas alcoólicas.

f) Provocou-me problemas em casa.

g) Meti-me à pancada ou destruí coisas.

h) Provocou-me um acidente, ferimentos, problemas com a polícia ou fui castigado na escola.

\section{Como se sente em relação ao que bebe?}

a) Sem problemas.

b) Posso controlar-me e impor limites a mim próprio.

c) Acho que posso controlar-me, mas os amigos influenciam- me com facilidade.

d) Tenho-me sentido mal comigo por beber.

e) Preciso que me ajudem para poder controlar-me.

f) Já tive de pedir ajuda ou que me tratassem por causa do que bebo.

\section{Como o acham os outros?}

a) Não sei, ou acham-me um bebedor normal para a minha idade.

b) Acham que quando bebo tenho tendência a negligenciar a minha família ou os amigos.

c) A família e/ou os amigos já me disseram para me controlar melhor, ou para cortar com o álcool.

d) A família e/ou os amigos já me aconselharam a procurar ajuda por causa do que bebo.

e) A família e/ou os amigos já alguma(s) vez(es) pediram ajuda para mim por causa da bebida. 